\title{
中药与肠道微环境相互作用研究进展
}

\author{
卷迪 ${ }^{1}$, 方明月 ${ }^{2}$, 李德利 ${ }^{2}$, 郑时奇 ${ }^{2}$, 乔姗姗 ${ }^{2}$, 王如峰 ${ }^{2}$, 杜力军 ${ }^{3 *}$ \\ 1. 北京中医药大学中药学院, 北京 102488 ; \\ 2. 北京中医药大学生命科学学院, 北京 102488 ; \\ 3. 清华大学生命科学学院, 北京 100084 \\ *联系人, E-mail: wangrufeng@tsinghua.org.cn; lijundu@mail.tsinghua.edu.cn \\ 收稿日期：2017-09-26; 接受日期：2017-12-02; 网络版发表日期：2018-03-22
}

摘要中药化学成分与肠道微环境的相互作用是中药发挥疗效的一个关键环节, 相关研究对阐明中药药效物质 及其作用机制至关重要, 其中涉及中药化学成分、肠道菌群和肠道细胞之间复杂的相互关系. 随着多学科理论和 技术的发展特别是本草基因组学学科概念的提出, 中药化学成分与肠道微环境的相互作用研究也由原来的化学 成分生物转化研究、菌群结构和免疫调控研究深入到基因水平, 其内容涉及结构和功能基因组、蛋白质组、转 录组、代谢组、表观基因组、宏基因组等理论与实验技术. 本文对该领域的研究进展进行了综述, 并对该领域未 来研究的发展方向进行了展望.

关键词中药, 肠道微环境, 相互作用

肠道微环境对人体的生理功能影响显著，在疾病 的发生、发展和消退过程中发挥重要作用。一般情况 下，肠道微环境保持相对稳定，这是其内部的肠道菌 群，营养和药物成分以及肠道细胞相互作用后，各方 面达到平衡的结果, 是矛盾各方的对立统一体. 这种 平衡状态的维持受矛盾各方力量此消彼长的影响, 一 旦某一方面, 如肠道菌群的结构发生明显的变化, 则 会对其他方面，如营养和药物成分以及肠道细胞产生 影响, 导致肠道微环境稳态被打破, 最终影响人体健 康. 同样, 通过人为地干预发生变化的某一方面, 如肠 道菌群的结构, 或者营养和药物成分的组成, 使其恢复 原来的状态，这又有可能造成矛盾的其他方面的逆向 变化, 导致肠道微环境恢复原来的状态. 中药的化学
成分进入人消化道后，与肠道微环境相互作用，引起 肠道微环境的变化. 正是由于中药引起的肠道微环境 的变化, 使其能够调节人体生理状态, 产生治疗疾病 的作用. 近年来, 在中药化学成分与肠道微环境的相 互作用方面进行了大量的研究, 随着多学科理论和技 术的发展特别是本草基因组学学科概念 ${ }^{[1]}$ 的提出, 中 药化学成分与肠道微环境的相互作用研究也逐渐深入 到基因水平. 这些研究结果为说明中药为什么有效的 问题开辟了新途径，也许是打开中药作用机制“黑箱” 的金钥匙.

\section{1 肠道微环境概述}

肠道微环境包括肠道菌群、肠道细胞和黏膜化学

引用格式: 卷迪, 方明月, 李德利, 等. 中药与肠道微环境相互作用研究进展. 中国科学: 生命科学, 2018, 48: 379-389

Geng D, Fang M Y, Li D L, et al. Research progress in terms of interaction between Chinese medicine components and intestinal microenvironment (in Chinese). Sci Sin Vitae, 2018, 48: 379-389, doi: 10.1360/N052017-00213 
屏障，是人体和外界进行物质、能量和信息交换的第 一道屏障, 也是饮食-药物-病原微生物等外界因素和 生理-病理状态下的人体交互作用的界面. 肠道菌群是 人肠道内的正常微生物, 人体内含有约 100 万亿个肠道 菌, 是人体自身细胞总数的 10 倍, 它所编码的基因是人

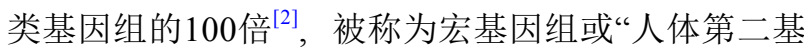
因组 ${ }^{n[3]}$. 肠道菌大约有1000 1150种，大部分集中于厚 壁菌门和拟杆菌门, 少数分布在变形菌门、放线菌 门、疮微菌门和梭杆菌门 ${ }^{[4]}$. 肠道菌群对人体生理的 主要功能有: (i) 通过自身酶类促进肠道吸收非消化 性营养物质 ${ }^{[5]}$; (ii) 促进免疫系统发育 ${ }^{[6]}$; (iii) 抵御病 原菌侵袭 ${ }^{[7]} ;$ (iv) 促进肠道血管生成 ${ }^{[8]} ;(\mathrm{v})$ 保护肠道 上皮免受损伤 ${ }^{[9]}$. 肠道细胞起源于隐窝处的肠道干细 胞，这些干细胞分化形成功能不同的肠道细胞. 成熟 的肠道细胞主要有 4 种类型: 吸收相关细胞、分泌细 胞、肠内分泌细胞和免疫相关细胞. 吸收相关细胞约 占肠上皮细胞的 $95 \%$, 负责吸收营养物质或药物. 分泌 细胞(杯状细胞)负责分泌黏蛋白形成黏液层，使肠道 上皮层与肠道菌群分离并抵御病原微生物 ${ }^{[10]}$. 肠内分 泌细胞分泌脑肠肽，包括胰高血糖素样肽 -1 、酪酪 肽、胆囊收缩素、葡萄糖依赖性胰岛素释放肽、5-羊圣 色胺和胃饥饯素等. 脑肠肽作用于脑肠轴, 调节吸收和 代谢、摄食行为、肠道蠕动、排空和渗透性等生理功 能 ${ }^{[1,12]}$. 免疫相关细胞(潘氏细胞)分布在隐窝底部, 产 生抗菌肽并调节肠道菌群，同时也产生生长因子维持 干细胞稳态 ${ }^{[13]}$. 黏膜化学屏障含有胃酸、黏液、胆 汁、糖蛋白以及消化酶等活性物质，具有保护肠道 细胞不受酸、碱侵蚀的作用，同时能够阻止致病菌 定植 ${ }^{[1416]}$.

健康的人体肠道微环境保持稳态，内部各因素维 持良性循环; 病理状态下稳态被打破，进而引发代谢 性疾病、免疫性疾病、神经系统疾病甚至癌症等多种 疾病 ${ }^{[17]}$. 肠道微环境稳态是微环境的外部因素(饮食、

药物、外来微生物)和内部因素(肠道菌群-黏膜和肠道 细胞)相互作用的结果, 而这些相互作用是由人体自身 基因和肠道菌群的基因所决定的.

\section{1 人体与肠道菌群的相互作用}

人体通过肠道细胞与肠道菌群相互作用, 肠道细 胞可直接影响或通过免疫系统调节肠道菌群的结构, 肠道菌群也可以通过代谢影响宿主正常生理状态和疾
病状态.

（1）宿主对肠道菌群的影响. 宿主对肠道菌群的 影响具有物种特异性, 并且宿主遗传变异也可以改变 肠道菌群的结构. 通过对比研究发现, 不同物种中定 植的肠道菌群的组成不同, 例如, 两种水螅 (Hydra vulgaris 和 H. oligactis)的菌群结构存在较大差异, H. vulgaris菌群结构较为丰富, 而H. oligactis则相对较少 ${ }^{[18]}$. 然而, 不同物种中的肠道菌群可以相互影响, 例如, 斑 马鱼(Danio rerio)和小鼠(Mus musculus)的肠道菌群可 相互定植, 改变各自的菌群结构 ${ }^{[19]}$. 宿主对肠道菌群的 影响与宿主的遗传物质有关，相同基因型的宿主的肠 道菌丰度更相似. Goodrich等人 ${ }^{[20]}$ 比较了英国416对双 胞胎超过 1000 份粪便样本的肠道菌组成, 发现同卵双 胞胎中克里斯滕森菌科(Christensenellaceae)肠道菌的 丰度相似. 目前甚至认为, 人的遗传物质是肠道菌群结 构的决定因素，通过对1126对英国双胞胎的全基因组 关联分析 (genome-wide association, GWAS)发现, 肠道 菌与宿主饮食偏好、代谢以及嗅觉相关基因相互关 联, 如乳糖酶基因(lactase gene, LCT)与人肠道中的双 歧杆菌存在关联性 ${ }^{[21]}$. 人类个体基因组的单核苷酸多 态性不但导致表型差异, 而且可造成个体肠道菌群结 构的差异, 目前已确定 58 个单核苷酸多态性位点的差 异与 33 类菌群丰度有关 ${ }^{[22]}$. 深入的基因转录产物研究 还发现, 人肠道中存在来源于IEC和Hopx ${ }^{+}$细胞的 miR$\mathrm{NA}$, 通过与肠道菌群相互作用而影响其生长 ${ }^{[23]}$.

宿主免疫在肠道菌群的调节中发挥介导作用，人 免疫相关基因通过表达抗菌肽影响肠道菌群. 例如, 潘氏细胞通过分泌抗菌肽和溶菌酶影响肠道菌群结 构，敲除肠道表皮细胞 I 型干扰素受体(IFNAR1)的基 因可促进肠道潘氏细胞的增殖, 而改变菌群结构又可 减弱肠道细胞的过量增殖 ${ }^{[24]}$. 由此可见, 肠道细胞增 殖也是肠道菌群和宿主相互作用的结果.

(2) 肠道菌群对宿主的影响. 肠道菌群既可以直 接影响宿主基因组的结构，也可以通过干预宿主的基 因表达而间接影响宿主基因组的功能. 肠道菌分泌的 毒素可诱发基因突变损伤宿主基因组，或通过细胞毒 性作用和干预细胞信号转导引发癌症。例如，带有聚 酮合成酶基因 $(p k s)$ 的大肠杆菌，可表达基因毒素colibactin蛋白, 引发真核细胞DNA双链损伤, 使小鼠肠道 上皮细胞组蛋白H2AX位点磷酸化 ${ }^{[25]}$. 又如, 在大肠杆 菌和空肠弯曲杆菌中发现的CDT (cytolethal distending 
toxin)借助细胞膜针定和内吞作用进入人肠道细胞, 通 过逆膜运输至细胞核造成DNA损伤 ${ }^{[26]}$. 某些肠道菌可 干预肠道细胞基因的表达，进而影响细胞功能. 例如, Salmonella typhimurium 可激活Wnt信号通路，短时间 内培养的小肠上皮细胞IEC-18与 $S$. typhimurium共培 养, 可提高 $W n t 2, W n t 2 b, W n t 3 a, W n t 7 b$ 和 $W n t 10 b$ 基因 表达水平1 6倍 ${ }^{[2730]}$. 肠道菌群通过对肠道干细胞或者 成熟肠道细胞的影响来维持肠道微环境稳态, 例如, 肠 道菌群可激活MyD88依赖的级联反应，增加黏膜分泌， 快速募集单核细胞至表皮隐窝干细胞周围，修复肠道 损伤 ${ }^{[31]}$.

此外，肠道菌群还可以通过调节宿主的生理状态 和营养吸收而间接影响宿主的基因状况 ${ }^{[32]}$. 它们中有 些可降解非消化性碳水化合物产生乙酸、丙酸和丁酸 等短链脂肪酸. 短链脂肪酸在肠道内为肠道细胞提供 能量, 同时促进细胞的生长, 维持肠道稳态. 研究发现, 人肠道菌群中有 9 个属为中国健康年轻人群所共有, 包 括厚壁菌门的Phascolarctobacterium, Roseburia, Blautia, Faecalibacterium, Clostridium, Subdoligranulum, Ruminococcus和Coprococcus属以及拟杆菌门的Bacteroides属. 这9个属的肠道菌都具有产生短链脂肪酸的 能力, 是对维持人体正常生理状态有益的共生菌 ${ }^{[33]}$. 对无菌(germ-free)小鼠的研究也发现，成年无菌小鼠 比相同年龄、相同性别的正常小鼠(出生后肠道菌群 定植)体重较轻 ${ }^{[34]}$.

\section{2 饮食对肠道微环境的影响}

饮食对肠道菌群有影响，不健康的高脂饮食可导 致肥胖, 其机制可能是高脂饮食导致肠道菌群结构改 变所致. 移植胖瘦不一致的双胞胎的肠道菌至无菌小 鼠后，发现移植肥胖个体的小鼠获得更多体重和脂 肪 ${ }^{[35]}$. 刘雪姬等人 ${ }^{[36]}$ 对控制饮食的小鼠肠道微生物菌 群结构信息进行了分析，发现高脂饮食的小鼠肠道中 乳酸杆菌、双歧杆菌及肠球菌的数量显著减少, 这可 能引发慢性炎症，导致肥胖的发生. 为从饮食角度改 善肠道菌群组成，Roberfroid等人 ${ }^{[37]}$ 提出益生元的概 念, 益生元是一类人体从食物中摄取但自身不可消化 的碳水化合物, 可被少数肠道菌(bifidobacteria和lactobacilli)利用，能够促进肠道菌的增殖和/或提高其活性， 对宿主健康起到有益的作用. 饮食还可以间接影响肠 道菌群，例如，饮食中的脂肪和蛋白质能促进胆汁酸
的分泌，胆汁酸对肠道菌群中不同菌种有抑制作用， 从而影响菌群结构 ${ }^{[38,39]}$.

饮食对肠道菌群的影响需要一个时间过程，长期 饮食习惯对肠道菌群的影响是决定性的. 然而, 肠道 菌群结构对不同饮食的响应时间不同，纤维性饮食和 脂类饮食在短时间内就可以引起肠道菌群的改变. Sonnenburg和Bäckhed ${ }^{[40]}$ 将植物性饮食和肉食性饮食 交替改变, 发现肠道菌群1 2天即可响应, 产生功能和 组成的变化.

\section{2 中药与肠道微环境相互作用研究}

与饮食一样，中药的化学成分也与肠道微环境发 生相互作用. 这主要体现在中药化学成分对肠道菌群 的结构和代谢, 以及肠道细胞功能的影响; 肠道菌群 对中药化学成分的结构修饰, 对肠道细胞功能的影响; 肠道细胞对中药化学成分的吸收和代谢, 对肠道菌群 代谢的影响等 ${ }^{[41]}$. 也就是中药化学成分、肠道菌群和 肠道细胞三者存在动态的相互作用关系(图1).

\section{1 中药对肠道微环境的作用}

中药化学成分可改变肠道菌群的结构并影响肠道 菌代谢, 也可以直接作用于肠道细胞, 影响肠道细胞的 数量和功能.

(1) 中药对肠道菌群的影响. 中药对肠道菌群有 双向调节效应, 一方面可促进有益菌的增殖, 另一方面 可抑制有害菌的水平, 即“扶正祛邪作用”. 补益类中药 可促进益生菌增殖, 这与其中所含多糖成分的类似于 益生元的作用有关. 补中益气汤中含有苷类、糖类物 质及多种微量元素, 能增加乳酸杆菌、双歧杆菌、枯 草芽狍杆菌的数量 ${ }^{[42]}$. 茯苓能显著提高双歧杆菌的水 平, 党参对肠道乳杆菌水平有提升作用 ${ }^{[43]}$, 含有党 参、获苓、白术等中药的复方也可增加乳杆菌、双歧 杆菌的数量, 减少肠球菌的数量 ${ }^{[44]}$. 清热解毒类中药多 具有抑菌活性，通过改善肠道菌群组成而利于肠道微 环境及宿主整体的健康. 张旭等人 ${ }^{[45]}$ 发现，小檗碱可 预防肥胖和胰岛素抵抗，其机制可能是通过改变肠道 菌群结构来减少机体外源性抗原并提高短链脂肪酸水 平, 从而缓解炎症. 体外厌氧培养肠道菌群后给予金银 花水提物，低浓度下可以促进双歧杆菌、乳酸杆菌的 生长, 而高浓度下则抑制这两种菌的生长 ${ }^{[46]}$. 


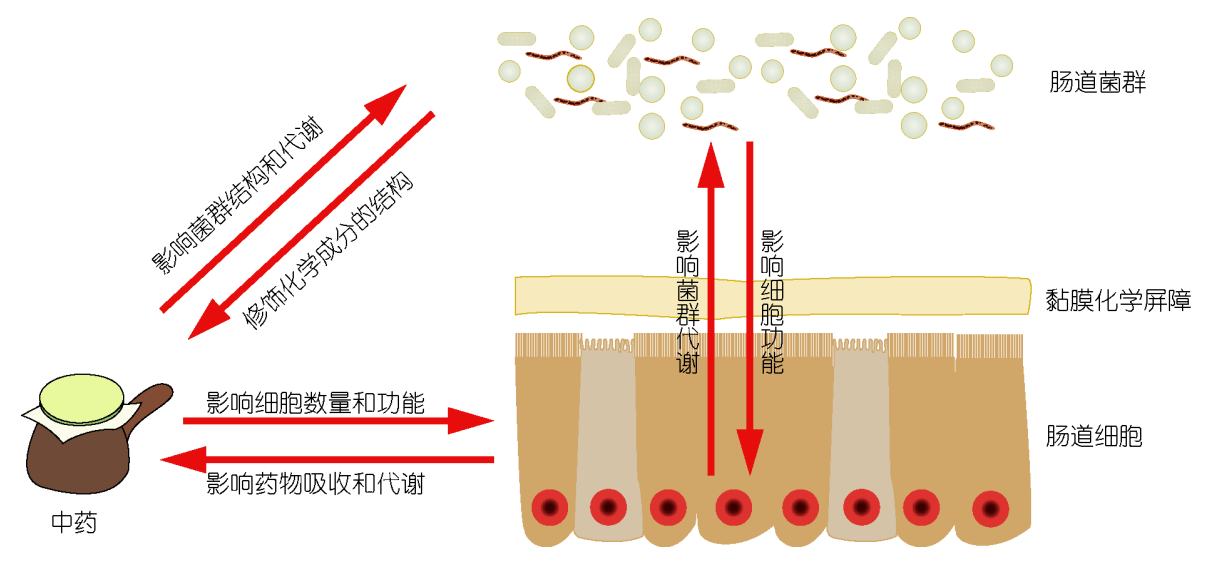

图 1 中药与肠道微环境相互作用模式图(网络版彩图)

中药不仅改善肠道菌群结构, 而且影响肠道菌自 身物质和能量代谢。在急性毒性剂量下，乌头碱导致 肠道菌内源性代谢谱发生改变 ${ }^{[4]}$. 四磨汤口服液则通 过提高肠道微生物分泌的纤维素酶和木聚糖酶的活 性, 恢复肠道微生态失调 ${ }^{[48]}$. 肠道菌自身代谢受中药的 影响后, 其代谢产物可以进一步作用于人体, 对人体生 理和病理状态产生影响，这也是中药作用机制的一个 很重要的环节, 但是目前相关研究较少.

（2）中药对肠道上皮细胞的影响. 中药既可以通 过调节肠道菌群代谢产物作用于肠道细胞, 又可以直 接作用于肠道细胞, 影响多种肠道细胞的数量及功能. 四君子汤提高胃大部分切除术后大鼠(Rattus norvegicus)肠上皮细胞增殖及代谢水平，减少细胞凋亡率 ${ }^{\left[{ }^{[9]}\right.}$. 在临床经验方肃肺润肠汤治疗药物依赖性便秘大鼠的 研究中, 发现大肠杯状细胞数量比模型组显著增加 ${ }^{[50]}$. 大承气汤对惣伤大鼠肠上皮细胞有保护作用，可减少 内毒素和肿瘤坏死因子的释放、减少肠组织的细胞凋 $亡^{[51]}$. 益气开秘方有一定的肠动力恢复作用, 其机制为 通过增加一氧化氮合成酶的表达促进NO释放, 对Cajal 细胞起保护作用 ${ }^{[52]}$.

某些中药化学成分可促进其他成分的吸收. 当归 与芳药配伍时，当归提取物可促进肠道对芳药苷的吸 收 ${ }^{[53]}$. 同样, 川芎配伍白芷时, 可促进白芷有效成分欧 前胡素与异欧前胡素的吸收，但是促进吸收的机制尚 不明确 ${ }^{[54]}$. 目前认为中药配伍促进化学成分的吸收是 通过调控肠道细胞的细胞色素P 450酶(cytochrome $\mathrm{P} 450, C Y P 450)$ 和P-糖蛋白(P-glycoprotein, $P-g p)$ 基因 的表达和功能. 例如, 芳香开窍类中药有促进肠道吸
收的功能, 有研究表明 3 种冰片对大鼠肠道P450酶和P$\mathrm{gp}$ 可产生不同的影响, 左旋龙脑和右旋龙脑不同程度 地提高肠道P450酶和P-gp的含量，而合成龙脑则显著 降低肠道中两者的含量 ${ }^{[55]}$. 甘草中的黄酮类成分可上 调Caco-2细胞P-gp蛋白的表达 ${ }^{[56]}$.

\section{2 肠道微环境对中药的作用}

肠道菌群通过自身酶系统对中药化学成分进行结 构修饰, 肠道细胞同样通过转运体蛋白和代谢酶影响 中药的代谢和吸收.

（1）肠道菌群对中药的影响. 肠道菌群自身代谢 酶系丰富, 对中药成分的结构修饰反应类型多样, 以水 解、氧化和还原反应为主, 同时存在异构化、氧氮转 化、聚合以及脱酰基化等特征性反应 ${ }^{[57]}$. 经过肠道菌 群的生物转化后, 中药化学成分结构发生改变, 药理 活性及毒性也随之变化. 例如, 虽然番泻叶有泻下作 用，但是其主要成分番泻苷并无泻下活性，只有被肠 道菌群转化为其代谢产物大黄酸后才表现出药理活 性 ${ }^{[58-61]}$. 在大鼠中进行的人参㿝苷代谢研究发现, 肠道 菌群中厚壁菌门、拟杆菌门和变形菌门数量明显较少 的高代谢率大鼠将人参㿝苷代谢成其苷元的效率明显 高于上述 3 个门菌种数量明显较少的低代谢率大鼠 ${ }^{[62]}$. 这不但说明肠内菌转化可以增强疗效, 而且也暗示肠 道菌群结构差异对中药代谢有重要影响. 同样, 肠内 菌转化也可减弱中药的疗效. 华蟾毒精和华蟾毒它灵 为中药蟾酥的主要活性成分, 经过肠道菌群转化, 原 形成分被去乙酰化, 药理活性明显降低 ${ }^{[63]}$. 另外, 肠道 菌群的转化作用也可降低或增加中药的毒性. 例如, 曾 
经普遍认为关木通和马自铃的肾毒性成分是马樂铃 酸, 其实, 经过系统研究后发现导致肾毒性的成分为马 皃铃酸的肠内菌转化产物马泉铃内酰胺 ${ }^{[64]}$. 肠道菌群 也介导中药不同成分之间的相互作用，当药药和甘草 $1: 1$ 配伍时，甘草抑制药药中苟药苷的转化; 而当药药 和甘草 $4: 1$ 配伍时，药药则显著抑制甘草中甘草苷的转 化，其机制可能是两种成分的共代谢途径一一去糖基 化均需要肠道菌群产生的 $\beta$-葡萄糖苷酶, 是该酶对莳 药苷和甘草苷竞争性催化的结果 ${ }^{[65]}$. 肠道菌群可对中 药的治疗作用产生直接或间接的影响, 因此, 有学者 提出需要研究宏基因组对药物治疗的影响 ${ }^{[66]}$.

（2）肠道细胞对中药的影响. 肠道细胞是药物吸 收的重要场所, 中药与肠道上皮细胞接触后通过主动 或被动转运进入细胞, 执行主动吸收功能的是转运蛋 白. 某些中药化学成分的生物利用度与两种不同功能 的转运蛋白有关, 即吸收转运体和外排转运体, 这些 化学成分吸收的最终结果由其与两种转运蛋白的亲和 力以及两种转运蛋白的表达量共同决定. 利用 Caco-2 细胞模型对金莲花中的黄酮类化合物的肠吸收试验表 明, 具有7-OH和2"-OH的黄酮类化合物诸如荭草素和 牡荆素是P-gp的底物, 当加入 P-gp抑制剂维拉帕米后, 可以促进这些化合物的吸收 ${ }^{[67]}$. 张海燕和平其能 ${ }^{[68]}$ 发 现，P-gp对灯戔花素的肠道细胞吸收没有影响，而多 药耐药相关蛋白MRP2可将吸收的灯盏花素外排至回 肠腔, 降低灯戞花素的生物利用度.

肠道细胞对中药影响的另一个重要方面是代谢, 尽管早在1970年就有肠道代谢的报道，但是普遍认为 药物肝代谢更为重要. 随着研究的不断深入, 发现肠 道细胞对药物的代谢率甚至有时高于肝代谢 ${ }^{[69]}$. 肠道 代谢酶中, 最主要的是执行氧化、还原和水解功能 的 I 相代谢酶P450酶系统，除此以外，还有尿苷二磷 酸葡萄糖醛酸转移酶(UDP-glucuronosyltransferase, $\mathrm{UGT}$ )、磺基转移酶、 $N$-乙酰基转移酶和谷胱甘肽转 移酶等 II 相代谢酶. 例如, 利用触皮素可被小肠上皮细 胞中的代谢酶甲基化 ${ }^{[70]}$; 白藜芦醇苷在胃肠道内转化 为白藜芦醇后, 在肠道细胞中发生葡萄糖醛酸化 ${ }^{[71]}$.

\section{3 中药与肠道微环境相互作用研究方法}

中药与肠道微环境的相互作用研究是传统医学、 现代生命科学和信息数据处理技术的结合. 其主要流
程中涉及化学方法、生物技术和生物信息学手段(图 2). 通过合理的试验设计并建立科学的数学模型, 对相 互作用的结果进行分析和预测将是深入研究的保证.

\section{1 体内外模型}

中药与肠道微环境相互作用研究的主要内容是中 药-肠道菌群-肠道细胞三者的联动关系, 建立相关联 的体内外模型尤为重要. 受限于肠道菌群的培养条件, 大多数肠道菌不能在体外培养, 而且实验室培养条件 不同, 其肠道菌群结构差异很大. 为了使体外培养的 肠道菌群具有多样性和稳定性, 需要可控的培养条件 和适合于更多菌种生长的培养基. Bollmann等人 ${ }^{[72]}$ 使 用扩散小室模拟定植环境, 培养不能在实验室条件下 培养的环境菌. Delaney等人 ${ }^{[73]}$ 应用高通量的厌氧培养 技术建立Methylobacterium extorquens的培养体系. 肠 道芯片微装置可体外模拟肠道微环境, 用于研究中药 与肠道微环境的相互作用 ${ }^{[74]}$. 动物模型可模拟人体, 用于血清药物化学和血清药理学研究. 模型动物一般 选用大鼠, 因为其个体小、饲养方便、繁殖快、质量 控制严格 ${ }^{[75]}$. 无菌动物是比较理想的模型动物, 目前 应用较为广泛的是无菌小鼠, 用于肠道菌群功能及药 物对肠道菌群影响的研究 ${ }^{[76,77]}$. 相比而言, 体外模型操 作相对简单, 条件易于控制, 影响因素较少, 可大规模 地复制且重复性好, 适合于代谢途径和作用机制研究; 而整体动物模型更接近人体内的真实情况, 是整体状 态下中药与肠道微环境作用的体现, 更符合中医药的 整体观. 根据研究目的和特点的不同, 选用不同的研 究模型，或者采用体内外模型相结合的方式，取长补 短, 相互印证, 有助于研究结果的准确性.

\section{2 代谢组学技术}

代谢组学(metabonomics)重点关注生物整体、器 官或组织的内源性代谢物质的代谢途径及其所受内在 或外在因素的影响及随时间变化的规律 ${ }^{[78]}$. 目前, 代谢 组学研究平台包括核磁共振谱(nuclear magnetic resonance, NMR)平台和质谱(mass spectrometry, MS)平台. 孙良平 ${ }^{[79]}$ 利用 GC-MS方法检测 2,4,6-三硝基苯磺酸诱 导损伤的大鼠黏膜组织, 发现了 10 种内源性差异代谢 物, 这为研究安肠汤对溃疡性结肠炎大鼠肠道微生态 的影响提供了重要依据. 多平台整合的代谢组学分析 可以克服单一分析技术对不同代谢物产生的歧视效 


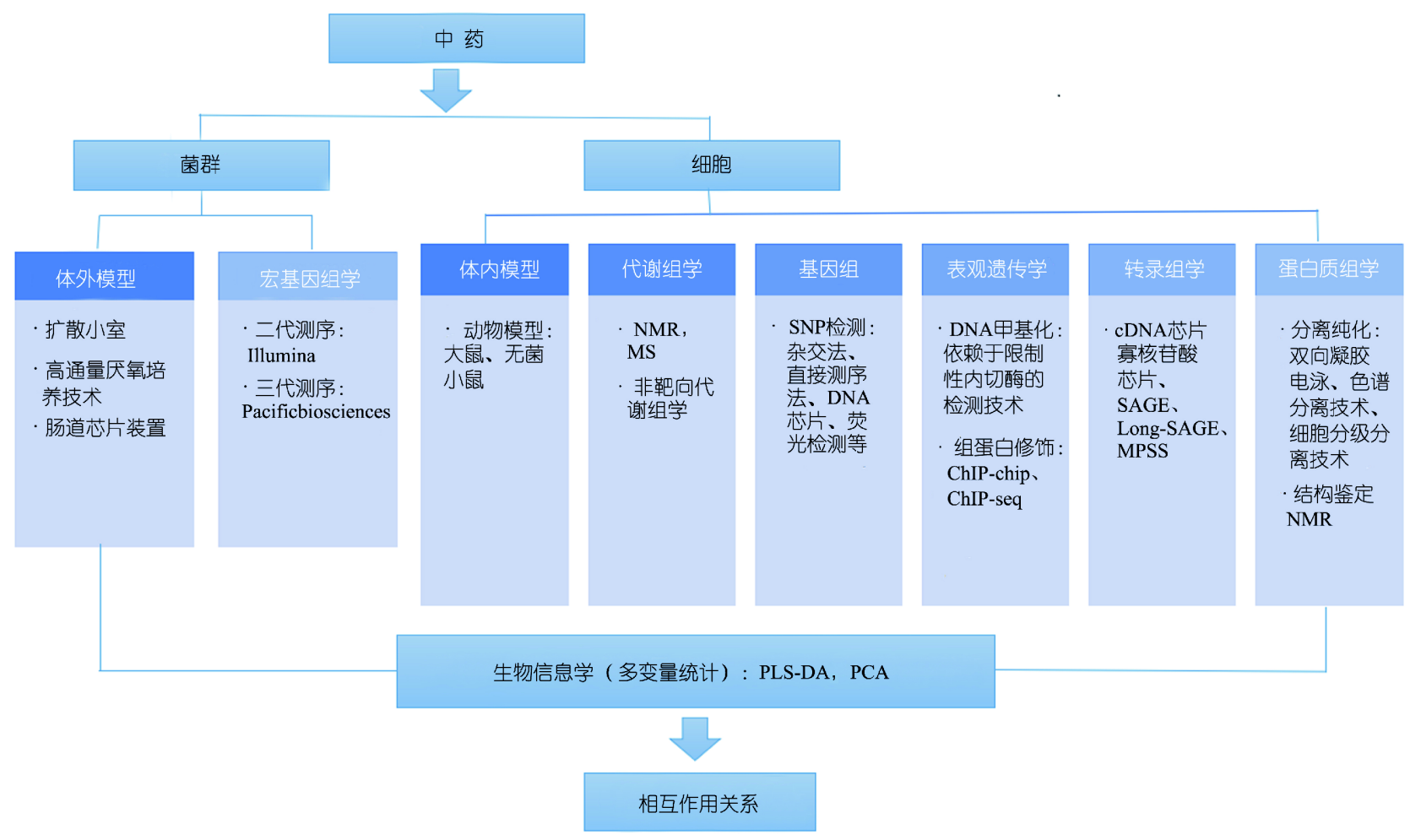

图 2 中药与肠道微环境相互作用的研究方法(网络版彩图)

应. 例如, 沈淑洁等人 ${ }^{[80]}$ 采用 ${ }^{1} \mathrm{H}-\mathrm{NMR}$ 和LC-MS技术分 析相结合的方法研究了金铃子散对足肿胀大鼠体内代 谢物的影响, 发现了18个炎症相关的差异代谢物. 针对 肠道微环境代谢产物的研究, 采用非靶向代谢组学更 为合适，通过分析多组样本的差异物质，找到肠道微 环境多因素联动对代谢产物的影响.

\section{3 肠道菌群宏基因组学技术}

宏基因组学(metagenomics)研究特定微生物群落 中所有微生物基因组总和及其功能，人肠道菌群宏基 因组学已经成为人体系统生物学研究的热点, 也为中 医药研究开辟了新领域 ${ }^{[81]}$. 利用宏基因组学研究肠道 菌群能够提供大量的基因信息，舒鹏 ${ }^{[82}$ 利用宏基因组 测序技术对小鼠肠道样品进行的研究发现，高脂饮食 减少拟杆菌门菌株, 同时增加变形菌门菌株含量, 说 明利用宏基因组测序技术可分析菌群构成，揭示疾病 治疗与肠道菌群的潜在联系. 随着新一代测序技术的 兴起，特别是随着边合成边测序的 454 , Illumina等二 代测序技术及能够直接测序和避免序列读长限制的三
代测序-单分子测序技术的出现, 使肠道菌群宏基因组 的高通量、大规模深度测序成为可能，再结合多变量 统计方法, 可以更直接地获得肠道微生物的组成和功 能信息 ${ }^{[83]}$ ．454测序技术于2005 2016年被使用，由于 其测序成本高和同聚物错误率高等缺陷 ${ }^{[84,85}$ 而被市场 淘汰. 第二个出现的测序平台是2006年Solexa公司(即 现在的Illumina公司)推出的基因组分析仪，采用可逆 性终结反应的原理进行桥式扩增，有效避免了对同聚 片段检测不灵敏的缺陷. 现有的技术平台有 GA/MiSeq/HiSeq, 具有通量高、成本低的优点 ${ }^{[6-88]}$. 有研究组 利用Illumina GA技术对不同健康状况的欧洲人的肠 道菌群进行了测序，得到了多于330万个基因，通过分 析发现几乎所有基因(99.1\%)都来源于细菌，其中包含 众多未知细菌 ${ }^{[89]}$. 其他的二代测序技术包括以塞核酸 连接反应测序的 $5500 \mathrm{xl} \mathrm{WSOLiD}^{[90]}$ 和基于半导体技术 和质子检测原理的Ion $\mathrm{PGM}^{[91]}$. 鉴于二代测序存在依 赖于模板扩增以及序列读长限制等缺点，近几年研发 出了三代测序——单分子测序技术，现仅有PacBio公 司的RS (real-time sequencing)系列测序仪得到商业化 应用 ${ }^{[92]}$. 


\section{4 基因组、表观遗传组及转录组学技术}

肠道微环境中的肠道细胞蕴涵着个体基因组的全 部信息, 基因组存在单核苷酸多态性(single nucleotide polymorphism，SNP)、个体表型差异、对药物或疾病 的易感性等都可能与 SNP有关，SNP检测包括区分 SNP特异位点和数据的检测分析方法 ${ }^{[33]}$, 前者有基于 杂交的方法、基于酶或PCR的方法、以构象为基础的 方法以及直接测序法; 后者则包括凝胶分析技术、苂 光检测技术、DNA芯片和质谱检测技术. 基因组是遗 传进化的结果, 但是, 个体基因组的表达还受到环境因 素的影响，同一个体因饮食习惯和肠道微环境的差异 其表观遗传修饰存在差异.

表观遗传修饰主要包括DNA甲基化和组蛋白修 饰. DNA甲基化检测方法分为依赖于限制性内切酶的 检测技术、依赖亚硫酸盐转换的检测技术和结合免疫 方法. 组蛋白修饰研究主要集中在组蛋白甲基化和乙 酰化上，目前主要采用染色质免疫共沉淀结合芯片的 方法(chromatin immunoprecipitation-chip, ChIP-chip) 和免疫共沉淀后测序技术(ChIP-seq $)^{[94]}$.

转录组学研究能够从整体水平上研究基因功能以 及基因结构, 揭示特定生物学过程以及疾病发生过程 中的分子机理. 目前研究转录组的方法包括基于杂交 技术的cDNA芯片和寡聚核苷酸芯片、基于 sanger测 序法的SAGE (serial analysis of gene expression), Long SAGE和MPSS (massively parallel signature sequencing)、基于二代测序技术的转录组测序(RNA-Seq) 等 ${ }^{[95]}$. 例如, Gosalbes等人 ${ }^{[96]}$ 利用454测序技术对来自 10 个健康志愿者的肠道微生物的cDNAs进行了测序, 然后通过宏基因组分析揭示了肠道微生物在健康机体 中的功能模式, 为研究健康机体肠道微生物的功能提 供了依据.

\section{5 蛋白质组学技术}

蛋白质作为人类生命活动的直接执行者，其在肠 道中扮演的角色至关重要. 蛋白质组学研究被认为是 连接基因组和临床应用的桥梁 ${ }^{[97]}$. 蛋白质组学研究技 术分为蛋白质分离纯化和结构鉴定两部分，这两部分 又分别采用不同的技术. 常用的蛋白质分离技术包括 双向凝胶电泳技术、色谱分离技术(利用分子量大 小、疏水性质、带电情况以及免疫亲和等差异)、细
胞分级分离技术等. 分离纯化的难点在于样品富集量 和蛋白质的化学性质，不同蛋白质在细胞中含量差别 很大, 并且蛋白质之间的物理化学性质差别也很大 ${ }^{[98]}$. 邓辉 ${ }^{[99]}$ 采用高分辨双向凝胶电泳从肠道亚健康便秘人 群结肠黏膜组织中分离鉴定出了 17 个蛋白，其中 10 个 蛋白表达上调，7个蛋白表达显著下调. 蛋白质的鉴定 目前主要采用质谱技术，首选是基质辅助激光解吸附 电离飞行时间质谱(matrix assisted laser desorption/ionization-time of flight, MALDI-TOF)技术, 它采用脉冲 式的离子化技术, 从固相标本中产生离子, 并在飞行管 中测定其分子量. 其次是电喷雾质谱(electrospray ionization-mass spectrometry, ESI-MS), 它采用连续离子化 的方法, 从液相中产生离子, 联合四极质谱或在飞行时 间检测器中检测其分子量.

\section{6 生物信息学技术}

中药与肠道微环境相互作用研究是多学科理论交 叉与多种技术的综合运用，在研究过程中可得到大量 的数据, 并且数据格式多样. 例如, 运用气相色谱-质 谱联用技术、高压液相色谱-质谱联用技术得到中药 的化学成分组信息, 以此作为肠道微环境的输入信息; 然后利用不同进化水平的动物模型，给予不同浓度的 化学成分组干预，并在不同时间点取样，得到中药与 肠道微环境相互作用的动态信息. 这些动态信息包括 中药代谢组学、宿主宏基因组学、宿主基因组学等动 态信息(转录组学、蛋白质组学), 作为中药与肠道微 环境相互作用的输出信息. 此外, 由于不同体质的个 体肠道微环境存在差异，依据中医对体质的分型构建 动物模型, 得到中药干预不同肠道微环境的输出信息.

数据处理采用多变量统计学方法, 如偏最小二乘 法-判别分析(partial least squares discrimination analysis, PLS-DA)、主成分分析(principal component analysis, PCA)等统计学方法, 找到多因素之间动态的关联 关系 ${ }^{[100]}$. 为了在时间轴上发现中药化学组学、宏基因 组学和宿主基因组学的动态变化规律, 采用图形化、 可视化方式可能有助于直观地呈现实验结果.

\section{4 中药与肠道微环境相互作用研究的意义 及展望}

肠道微环境是人体系统中的一个次级开放系统, 肠道菌群和肠道细胞间存在相互作用的关系, 具有“整 


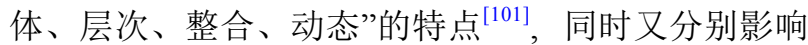
人体其他系统，通过层次之间、网络之间、系统之间 的整合和联系建立大小网络，而不同网络之间的信息 整合和传递使基因或蛋白质产生出最终的生物学功 能. 肠道微环境还是一个功能性的概念, 为中药作用机 制的研究提供了新思路和新靶标。中药处方“因人而 异”，这正好与肠道微环境的个体差异相契合. 但是这 种个体差异也增大了中药与肠道微环境相互作用研究 的难度, 需要能够整合复杂系统的有效研究手段才能 科学地阐释其作用规律.

近年来飞速发展的一些新学科和新技术, 如系统
生物学、网络药理学理论以及组学和生物信息学技术 等为解决上述问题提供了强有力的支持. 随着这些新 理论和新技术的不断成熟和应用，中药与肠道微环境 相互作用的研究将不断深入, 将会从简单的菌群结构 测定向系统探讨肠内菌的代谢变化，从定性描述向定 量建模，从局部静态揭示向整体动态分析，从机理阐 明向整体调控等方向发展. 总之，在大数据应用日益 广泛的今天, 中药化学成分、肠道微环境和人体被作 为一个有机的整体进行研究，这既符合中医的整体化 观念，又借鉴了现代自然科学的发展成果，对中医药 现代化的进程必将起到推动作用.

\section{参考文献}

1 陈士林, 宋经元. 本草基因组学. 中国中药杂志, 2016, 41: 3881-3889

2 Ley R E, Peterson D A, Gordon J I. Ecological and evolutionary forces shaping microbial diversity in the human intestine. Cell, 2006, 124: 837848

3 肖水明, 白瑞, 张小燕. 中药体内代谢基因组研究. 中国中药杂志, 2016, 41: 4103-4111

4 Eckburg P B, Bik E M, Bernstein C N, et al. Diversity of the human intestinal microbial flora. Science, 2005, 308: 1635-1638

5 Hooper L V, Midtvedt T, Gordon J I. How host-microbial interactions shape the nutrient environment of the mammalian intestine. Annu Rev Nutr, 2002, 22: 283-307

6 Hill D A, Artis D. Intestinal bacteria and the regulation of immune cell homeostasis. Annu Rev Immunol, 2010, 28: 623-667

7 Vollaard E J, Clasener H A. Colonization resistance. Antimicrobial Agents Chemother, 1994, 38: 409-414

8 Stappenbeck T S, Hooper L V, Gordon J I. Nonlinear partial differential equations and applications: developmental regulation of intestinal angiogenesis by indigenous microbes via Paneth cells. Proc Natl Acad Sci USA, 2002, 99: 15451-15455

9 Rakoff-Nahoum S, Paglino J, Eslami-Varzaneh F, et al. Recognition of commensal microflora by Toll-like receptors is required for intestinal homeostasis. Cell, 2004, 118: 229-241

10 刘玥宏, 丁悦, 徐敬东. 肠道杯状细胞及其功能研究进展. 世界华人消化杂志, 2017, 25: 1279-1286

11 Mace O J, Tehan B, Marshall F. Pharmacology and physiology of gastrointestinal enteroendocrine cells. Pharmacol Res Perspect, 2015, 3: $\mathrm{e} 00155$

12 Efeyan A, Comb W C, Sabatini D M. Nutrient-sensing mechanisms and pathways. Nature, 2015, 517: 302-310

13 龙振昼, 聂青和. 潘氏细胞及其抗微生物肽与肠道黏膜免疫. 世界华人消化杂志, 2017, (13): 209-219

14 于泳, 郑鹏远. 肠道细菌、肠黏膜屏障与肠功能障碍. 胃肠病学和肝病学杂志, 2013, 22: 121-122

15 胡红莲, 高民. 肠道屏障功能及其评价指标的研究进展. 中国畜牧杂志, 2012, 48: 78-82

16 朱强, 刘华光. 肠道微生态系统与常见消化系统疾病——肠道微生态系统与肠道黏膜屏障. 山东医药, 2011, 51: 100

17 Guarner F, Malagelada J R. Gut flora in health and disease. Lancet, 2003, 361: 512-519

18 Fraune S, Bosch T C G. Long-term maintenance of species-specific bacterial microbiota in the basal metazoan Hydra. Proc Natl Acad Sci USA, 2007, 104: 13146-13151

19 Rawls J F, Mahowald M A, Ley R E, et al. Reciprocal gut microbiota transplants from zebrafish and mice to germ-free recipients reveal host habitat selection. Cell, 2006, 127: 423-433

20 Goodrich J K, Waters J L, Poole A C, et al. Human genetics shape the gut microbiome. Cell, 2014, 159: 789-799

21 Goodrich J K, Davenport E R, Beaumont M, et al. Genetic determinants of the gut microbiome in UK twins. Cell Host Microbe, 2016, 19: 731743

22 Turpin W, Espin-Garcia O, Xu W, et al. Association of host genome with intestinal microbial composition in a large healthy cohort. Nat Genet, 
2016, 48: 1413-1417

23 Liu S, da Cunha A P, Rezende R M, et al. The host shapes the gut microbiota via fecal microRNA. Cell Host Microbe, 2016, 19: 32-43

24 Tschurtschenthaler M, Wang J, Fricke C, et al. Type I interferon signalling in the intestinal epithelium affects Paneth cells, microbial ecology and epithelial regeneration. Gut, 2014, 63: 1921-1931

25 Arthur J C, Perez-Chanona E, Mühlbauer M, et al. Intestinal inflammation targets cancer-inducing activity of the microbiota. Science, 2012, 338: $120-123$

26 Guerra L, Nemec K N, Massey S, et al. A novel mode of translocation for cytolethal distending toxin. BBA-Mol Cell Res, 2009, 1793: 489-495

27 Sun J, Hobert M E, Rao A S, et al. Bacterial activation of beta-catenin signaling in human epithelia. Am J Physiol-Gastr L, 2004, 287: G220G227

28 Sun J, Hobert M E, Duan Y, et al. Crosstalk between NF-kappaB and beta-catenin pathways in bacterial-colonized intestinal epithelial cells. Am J Physiol-Gastr L, 2005, 289: G129-G137

29 Duan Y, Liao A P, Kuppireddi S, et al. $\beta$-Catenin activity negatively regulates bacteria-induced inflammation. Lab Invest, 2007, 87: 613-624

30 Ye Z, Petrof E O, Boone D, et al. Salmonella effector AvrA regulation of colonic epithelial cell inflammation by deubiquitination. Am J Pathol, 2007, 171: 882-892

31 Skoczek D A, Walczysko P, Horn N, et al. Luminal microbes promote monocyte-stem cell interactions across a healthy colonic epithelium. J Immunol, 2014, 193: 439-451

32 Bäckhed F, Ley R E, Sonnenburg J L, et al. Host-bacterial mutualism in the human intestine. Science, 2005, 307: 1915-1920

33 Zhang J, Guo Z, Xue Z, et al. A phylo-functional core of gut microbiota in healthy young Chinese cohorts across lifestyles, geography and ethnicities. ISME J, 2015, 9: 1979-1990

34 Bäckhed F, Ding H, Wang T, et al. The gut microbiota as an environmental factor that regulates fat storage. Proc Natl Acad Sci USA, 2004, 101: $15718-15723$

35 Ridaura V K, Faith J J, Rey F E, et al. Gut microbiota from twins discordant for obesity modulate metabolism in mice. Science, 2013, 341: 1241214

36 刘雪姬, 陈庆森, 闩亚丽. 高脂饮食对小鼠肠道菌群的影响. 食品科学, 2011, 32: 306-311

37 Roberfroid M, Gibson G R, Hoyles L, et al. Prebiotic effects: metabolic and health benefits. Br J Nutr, 2010, 104: S1-S63

38 Begley M, Gahan C G M, Hill C. The interaction between bacteria and bile. FEMS Microbiol Rev, 2005, 29: 625-651

39 Kurdi P, Kawanishi K, Mizutani K, et al. Mechanism of growth inhibition by free bile acids in lactobacilli and bifidobacteria. J Bacteriol, 2006, 188: 1979-1986

40 Sonnenburg J L, Bäckhed F. Diet-microbiota interactions as moderators of human metabolism. Nature, 2016, 535: 56-64

$41 \mathrm{Xu}$ J, Chen H B, Li S L. Understanding the molecular mechanisms of the interplay between herbal medicines and gut microbiota. Med Res Rev, 2017, 37: 1-46

42 冯兴忠, 张娅南, 姜欣, 等. 加味补中益气汤促进肠道益生菌生长的实验研究. 中国微生态学杂志, 2008, 20: 159-160

43 宋克玉, 江振友, 严群超, 等. 党参及获苓对小鼠肠道菌群调节作用的实验研究. 中国临床药理学杂志, 2011, 27: 142-145

44 陈琛, 江振友, 宋克玉, 等. 中草药对小鼠肠道菌群影响的实验研究. 中国微生态学杂志, 2011, 23: 15-17

45 Zhang X, Zhao Y, Zhang M, et al. Structural changes of gut microbiota during berberine-mediated prevention of obesity and insulin resistance in high-fat diet-fed rats. PLoS ONE, 2012, 7: e42529

46 由域辰, 黎海芪, 刘明芳. 金银花水提物对双歧杆菌与乳酸杆菌体外生长的影响. 时珍国医国药, 2008, 19: 1131-1133

47 王旭涁, 曹瑞丽, 刘巧, 等. 乌头碱对大鼠粪样代谢谱影响的代谢组学研究. 中国医药导报, 2013, 10: 18-21 李丹丹, 肖新云, 赵先平, 等. 四磨汤口服液对脾虚便秘小鼠肠道微生物及酶活性的影响. 中国微生态学杂志, 2015, 27: 135-138 冯瑨, 黄建平, 胡俊波, 等. 肠内营养合早期中药干预对胃大部切除大鼠肠道黏膜细胞更新状态的影响. 上海中医药杂志, 2008, 42: 68-70 高健群, 巫全胜, 钱宁, 等. 肃肺润肠汤对药物依赖性便秘大鼠大肠杯状细胞、5-HT变化的实验研究. 时珍国医国药, 2010, 21: 1814-1815 王银山, 苏雅莉, 吴云虎, 等. 大承气汤对汮伤大鼠肠黏膜上皮细胞超微结构变化的影响. 中华中医药学刊, 2009, 27: 1768-1770 董艳, 何春梅, 陆金根. 益气开秘方调控肠道Cajal细胞NO-cGMP-PKG通路的实验研究. 上海中医药大学学报, 2013, 27: 82-86 易军, 吴秋平. 当归提取物等不同吸收促进剂对药药苷大鼠十二指肠的吸收促进作用. 时珍国医国药, 2014, 25: 2136-2138 张风玲, 黄燕芬, 易军. 川芎提取物等口服吸收促进剂对白芷在大鼠肠道吸收的作用. 广东药学院学报, 2015, 31: 300-304 雷玲, 白䈗璐, 胡竟一, 等. 三种冰片对大鼠肠道细胞色素P450和糖蛋白P-gp的影响. 中药药理与临床, 2016, 32: 83-87 
56 彭燕, 谭晓斌, 贾晓斌. 甘草黄酮类成分对Caco-2细胞P-糖蛋白功能和表达的影响. 中草药, 2013, 44: 2703-2709

57 Wang R F, Yuan M, Yang X B, et al. Intestinal bacterial transformation-a nonnegligible part of Chinese medicine research. J Asian Nat Products Res, 2013, 15: 532-549

58 Hattori M, Namba T, Akao T, et al. Metabolism of sennosides by human intestinal bacteria. Planta Med, 1980, 36: 172-179

59 Hattori M, Kim G, Motoike S, et al. Metabolism of sennosides by intestinal flora. Chem Pharm Bull, 1982, 30: 1338-1346

60 Sasaki K, Yamauchi K, Kuwano S. Metabolic activation of sennoside A in mice. Planta Med, 1979, 37: 370-378

61 Yamauchi K, Shinano K, Nakajima K, et al. Metabolic activation of sennoside C in mice: synergistic action of anthrones. J Pharmacy Pharmacol, 1992, 44: 973-976

62 Dong W W, Xuan F L, Zhong F L, et al. Comparative analysis of the rats' gut microbiota composition in animals with different ginsenosides metabolizing activity. J Agric Food Chem, 2017, 65: 327-337

63 Yang X, Xing Z, Cui J, et al. Studies on the metabolism of cinobufagin and cinobufotalin by human intestinal bacteria. J Beijing Med Univ, 2001, 33: 199-204

64 杨秀伟, 郝美荣, 服部征雄. 中药成分代谢分析. 北京: 中国医药科技出版社, 2003. 42-45, 689-690

$65 \mathrm{Xu} \mathrm{C} \mathrm{H}$, Wang P, Wang Y, et al. Pharmacokinetic comparisons of two different combinations of Shaoyao-Gancao Decoction in rats: competing mechanisms between paeoniflorin and glycyrrhetinic acid. J Ethnopharmacol, 2013, 149: 443-452

66 Haiser H J, Turnbaugh P J. Is it time for a metagenomic basis of therapeutics? Science, 2012, 336: 1253-1255

67 Liu L, Guo L, Zhao C, et al. Characterization of the intestinal absorption of seven flavonoids from the flowers of Trollius chinensis using the Caco-2 cell monolayer model. PLoS ONE, 2015, 10: e0119263

68 张海燕, 平其能. 药物转运蛋白对灯盏花素小肠吸收的影响. 中国药科大学学报, 2007, 38: 60-64

69 van de Kerkhof E G, Ungell A L B, Sjöberg A K, et al. Innovative methods to study human intestinal drug metabolism in vitro: precision-cut slices compared with ussing chamber preparations. Drug Metab Disposit, 2006, 34: 1893-1902

70 李素云, 李峥, 李敬来, 等. 葪皮素及其糖苷衍生物在Caco-2单层细胞上的吸收特征. 营养学报, 2012, 34: 358-361

71 Henry C, Vitrac X, Decendit A, et al. Cellular uptake and efflux of trans-piceid and its aglycone trans-resveratrol on the apical membrane of human intestinal Caco-2 cells. J Agric Food Chem, 2005, 53: 798-803

72 Bollmann A, Lewis K, Epstein S S. Incubation of environmental samples in a diffusion chamber increases the diversity of recovered isolates. Appl Environ Microbiol, 2007, 73: 6386-6390

73 Delaney N F, Kaczmarek M E, Ward L M, et al. Development of an optimized medium, strain and high-throughput culturing methods for Methylobacterium extorquens. PLoS ONE, 2013, 8: e62957

$74 \mathrm{Kim} \mathrm{H} \mathrm{J,} \mathrm{Li} \mathrm{H,} \mathrm{Collins} \mathrm{J} \mathrm{J,} \mathrm{et} \mathrm{al.} \mathrm{Contributions} \mathrm{of} \mathrm{microbiome} \mathrm{and} \mathrm{mechanical} \mathrm{deformation} \mathrm{to} \mathrm{intestinal} \mathrm{bacterial} \mathrm{overgrowth} \mathrm{and} \mathrm{inflammation}$ in a human gut-on-a-chip. Proc Natl Acad Sci USA, 2016, 113: E7-E15

75 王喜军. 中药及中药复方的血清药物化学研究. 世界科学技术: 中药现代化, 2002, 4: 1-4

76 Faith J J, McNulty N P, Rey F E, et al. Predicting a human gut microbiota's response to diet in gnotobiotic mice. Science, 2011, 333: 101-104

77 Grover M, Kashyap P C. Germ-free mice as a model to study effect of gut microbiota on host physiology. Neurogastroenterol Motil, 2014, 26: 745-748

78 贾伟. 医学代谢组学. 上海: 上海科学技术出版社, 2011. 1-3

79 孙平良. 基于代谢组学的溃疡性结肠炎大鼠肠道微生态变化及安肠汤干预机制研究. 广西: 广西医科大学, 2017

80 沈淑洁, 水素芳, 肖炳坤, 等. 基于1H-NMR及LC-MS技术研究金铃子散对炎症大鼠模型调节机制的影响. 中国中药杂志, 2017, 42: 363369

81 张旭, 赵宇峰, 胡义扬, 等. 基于功能元基因组学的人体系统生物学新方法: 中医药现代化的契机. 世界科学技术: 中医药现代化, 2011, 13: 202-212

82 舒鹏. 宏基因组测序技术在肠道菌群研究和临床样本检测中的应用. 合肥: 安徽医科大学, 2016

83 Wang W L, Xu S Y, Ren Z G, et al. Application of metagenomics in the human gut microbiome. World J Gastroenterol, 2015, 21: 803-814 Qin J, Li R, Raes J, et al. A human gut microbial gene catalogue established by metagenomic sequencing. Nature, 2010, 464: 59-65 Gilles A, Meglécz E, Pech N, et al. Accuracy and quality assessment of 454 GS-FLX Titanium pyrosequencing. BMC Genomics, 2011, 12: 245 
87 Jiang Z, Li P, Van Nostrand J D, et al. Microbial communities and arsenic biogeochemistry at the outflow of an alkaline sulfide-rich hot spring. Sci Rep, 2016, 6: 25262

88 Liu L, Li Y H, Li S L, et al. Comparison of next-generation sequencing systems. Biomed Res Int, 2012, 2012: e251364

89 王兴春, 杨致荣, 王敏, 等. 高通量测序技术及其应用. 中国生物工程杂志, 2012, 32: 109-114

90 Loman N J, Constantinidou C, Chan J Z M, et al. High-throughput bacterial genome sequencing: an embarrassment of choice, a world of opportunity. Nat Rev Micro, 2012, 10: 599-606

91 Spanu P D, Abbott J C, Amselem J, et al. Genome expansion and gene loss in powdery mildew fungi reveal tradeoffs in extreme parasitism. Science, 2010, 330: 1543-1546

92 乌日拉嘎, 徐海燕, 冯淑贞, 等. 测序技术的研究进展及三代测序的应用. 中国乳品工业, 2016, 44: 33-37

93 高秀丽, 景奉香, 杨剑波, 等. 单核苷酸多态性检测分析技术. 遗传, 2005, 27: 110-122

94 郑小国, 陈亮, 楼巧君, 等. 表观遗传学研究方法进展. 生物技术通报, 2011, 27: 63-71

95 侯志伟, 王望, 高宏, 等. dRNA-seq原理及其在原核生物转录组学研究中的应用. 遗传, 2013, 35: 983-991

96 Gosalbes M J, Durbán A, Pignatelli M, et al. Metatranscriptomic approach to analyze the functional human gut microbiota. PLoS ONE, 2011, 6: e17447

97 申定珠, 蔡震峰, 李家邦, 等. 蛋白质组学及其在消化系统疾病中的应用. 医学综述, 2006, 12: 52-55

98 夏其昌, 曾嵘. 蛋白质化学与蛋白质组学. 北京: 科学出版社, 2004. 233-240

99 邓辉. 肠道亚健康人群结肠黏膜蛋白质组学研究. 长沙: 中南大学, 2011

100 王文佶, 张秋菊, 曲思杨, 等. 动态代谢组学数据分析方法介绍. 中国卫生统计, 2016, 33: 1075-1078

101 陈海彬, 程海波, 卢伟, 等. 系统生物学是中西医结合的桥梁. 中国中西医结合杂志, 2013, 33: 119-124

\title{
Research progress in terms of interaction between Chinese medicine components and intestinal microenvironment
}

\author{
GENG Di ${ }^{1}$, FANG MingYue ${ }^{2}$, LI DeLi ${ }^{2}$, ZHENG ShiQi ${ }^{2}$, QIAO ShanShan $^{2}$, WANG RuFeng $^{2} \&$ \\ DU LiJun ${ }^{3}$
}

\begin{abstract}
1 School of Chinese Materia Medica, Beijing University of Chinese Medicine, Beijing 102488, China; 2 School of Life Sciences, Beijing University of Chinese Medicine, Beijing 102488, China;

3 School of Life Sciences, Tsinghua University, Beijing 100084, China
\end{abstract}

Interactions between the components of traditional Chinese medicine (TCM) and the intestinal microenvironment are a key to TCM effectiveness. Studies investigating the complex relationship among TCM components, intestinal microenvironment, and enterocytes are vital for elucidating the effective substances of TCM and the mechanisms underlying their activities. With the development of theories and techniques across multiple disciplines, particularly the emergence of herb genomics, investigation into the interactions between TCM components and intestinal microenvironment has evolved from the biotransformation of components and regulation of flora structure and immunity into a study at the gene level, which involves structural and functional genomics, proteomics, transcriptomics, metabolomics, epigenomics, and metagenomics. This review summarizes the research progress in terms of the scope of TCM and proposes areas for future studies.

\section{Chinese medicine, intestinal microenvironment, interaction}

doi: $10.1360 / \mathrm{N} 052017-00213$ 